\section{First year of the thoracic triumvirate}

\author{
Nicholas Hart, ${ }^{1}$ Gisli Jenkins, ${ }^{2}$ Alan Robert Smyth ${ }^{3}$
}

When we accepted the prestigious role of Joint Editors-in-Chief of Thorax 1 year ago we were fully aware of the enormous responsibility that came with the role as well as the challenges that we would face as the editorial team. We were wholly cognisant that we were representing more than just a journal, but indeed we were representing an important national body, the British Thoracic Society (BTS). Our key aims were to focus on originality, excellence and rigour in respiratory, sleep and critical care medicine to ensure that Thorax delivers interesting, educational and impactful content to all its readers, most importantly BTS members. We were tasked with building up still further the publication metrics. In the last year, our top read articles have included the pulmonary nodule guidelines, ${ }^{1}$ read over 9000 times and cited 17 times, original research looking at the use of venous blood gas analysis in COPD, ${ }^{2}$ read over 6700 times, clinical trial data from the Lebrikizumab trials, ${ }^{3}$ read over 5000 times and cited 16 times, basic science studies investigating the role of Toll-like receptor 7 in regulating responses to rhinovirus, ${ }^{4}$ read over 3000 times and cited 4 times, and a network meta-analysis investigating combinations of LABA/ LAMAs in COPD, ${ }^{5}$ read nearly 2000 times. We have published important consensus statements for cystic fibrosis, including non-tuberculous mycobacteria, ${ }^{6}$ read 3348 times, and on mental health, ${ }^{7}$ read 3786 times. We believe these studies show that we are capturing the breadth of respiratory research from bench-to-bedside and onto healthcare implementation.

In line with our strategic aims, we have made substantial changes to Thorax, some of which have led to immediate effects while others are likely to take longer to reveal their benefits. We have increased the word limit of our original research articles (now 3500 words) to permit more methodological detail and more results

\footnotetext{
'Lane Fox Respiratory Service, Guy's \& St Thomas' NHS Foundation Trust, London, UK; ${ }^{2}$ Centre for Respiratory Research, University of Nottingham, Nottingham, UK; ${ }^{3}$ Division of Child Health, Obstetrics \& Gynaecology, University of Nottingham, Nottingham, UK

Correspondence to Dr Gisli Jenkins, Centre for Respiratory Research, University of Nottingham, Nottingham NG51PB, UK; gisli.jenkins@nottingham.ac.
} uk that we hope will encourage the submission of more comprehensive studies. We have substantially altered our letters and correspondence sections. To promote our on-line presence and encourage real-time discussion of manuscripts, we have removed the correspondence section from the print journal and are working on developing online discussion forum to encourage people to submit such correspondence. To promote the rapid publication of novel, important observations that may not have a fully developed prospective dataset or lack detailed mechanistic studies, we have promoted the Research Letters section with great success, with a $90 \%$ increase in submissions and a $183 \%$ increase in publications in this category. We will continue to promote the Research Letter as a way to rapidly publish important observations, which we hope will stimulate follow-up studies leading to high quality original manuscripts.

To facilitate the review process for authors and reviewers, we have made a number of substantial changes. We require that all clinical trials and systematic reviews are pre-registered before they are undertaken so that the performance of the study and the results can be assessed in line with the published protocol. Deviations from the protocol are likely to lead to rejection unless there is a clear and approved rationale for change. The submitted manuscript must include the appropriate CONSORT, PRISMA, and for basic science in vivo studies, ARRIVE checklists to ensure the studies conform to reporting guidelines. We believe these changes will drive up quality and speed up the review process. It is worth authors remembering that not only is research an evolving process, but so is publication and we regularly update the instructions to authors which contain important information, which, if not adhered to, may lead to immediate rejection.

Another major initiative has been to have all submitted manuscripts that include data assessed by our statistical editors. This has increased the workload of these editors considerably and to mitigate this, we have expanded the pool of editors considerably. Their efforts have been exceptional and their reviews are crucial. We expect that their comments are taken exceptionally seriously, as failure to address them is likely to lead to rejection of the manuscript after review.
As Editors, we are aware that decisions made on manuscripts are, in large part, subjective. Given the low acceptance rates of Thorax, which have remained at $7 \%$ over the last 12 months, we accept that many manuscripts of good quality will be rejected; however, our aim is to publish the very best of the submitted manuscripts. We have increased the immediate reject rate, which now stands at $60 \%$, to speed up the publication process, as we believe a high instant reject rate gives authors the best chance of getting their work published elsewhere in a timely fashion. The result of this has led to a reduction in our time to first decision, which is now only 17 days. We are pleased to see that this policy has not deterred authors, indeed submission have increased by nearly 5\% and submission and acceptance from North America have increased by $9 \%$ and $64 \%$, respectively.

Overall, we believe our first year has been successfully facilitated by the excellent submissions we have received, the timely and thorough reviews that have been performed and the outstanding efforts of the associate and statistical editors. Most importantly, as a triumvirate, we have adopted a position of shared responsibility without any descent into civil war, which we believe is more successful than some previous triumvirates throughout history.

Twitter Follow Nicholas Hart at @NickHartThorax, Gisli Jenkins at @IPFdoc and Alan Robert Smyth at @AlanRSmyth.

Competing interests None declared.

Provenance and peer review Commissioned; internally peer reviewed.

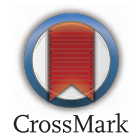

To cite Hart N, Jenkins G, Smyth AR. Thorax 2016;71:579-580.

Thorax 2016;71:579-580.

doi:10.1136/thoraxjnl-2016-208959

\section{REFERENCES}

1 Callister ME, Baldwin DR, Akram AR, et al. British Thoracic Society guidelines for the investigation and management of pulmonary nodules. Thorax 2015;70 (Suppl 2):ii1-54.

2 McKeever TM, Hearson G, Housley G, et al. Using venous blood gas analysis in the assessment of COPD exacerbations: a prospective cohort study. Thorax 2016;71:210-15.

3 Hanania NA, Noonan M, Corren J, et al. Lebrikizumab in moderate-to-severe asthma: pooled data from two randomised placebo-controlled studies. Thorax 2015;70:748-56

4 Hatchwell L, Collison A, Girkin J, et al. Toll-like receptor 7 governs interferon and inflammatory responses to rhinovirus and is suppressed by 


\section{Editorial}

IL-5-induced lung eosinophilia. Thorax 2015;70:854-61.

5 Oba Y, Sarva ST, Dias S. Efficacy and safety of long-acting $\beta$-agonist/long-acting muscarinic antagonist combinations in COPD: a network meta-analysis. Thorax 2016;71:15-25.
6 Floto RA, Olivier KN, Saiman L, et al. US Cystic Fibrosis Foundation and European Cystic Fibrosis Society consensus recommendations for the management of non-tuberculous mycobacteria in individuals with cystic fibrosis. Thorax 2016;71(Suppl 1):i1-22.
7 Quittner AL, Abbott J, Georgiopoulos AM, et al. International Committee on Mental Health in Cystic Fibrosis: Cystic Fibrosis Foundation and European Cystic Fibrosis Society consensus statements for screening and treating depression and anxiety. Thorax 2016;71:26-34. 